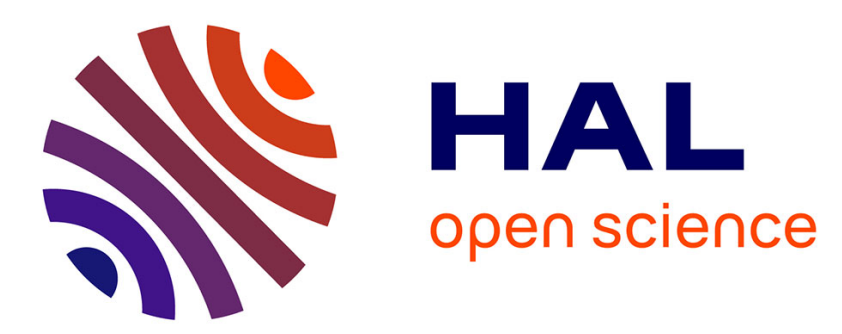

\title{
A Numerical Study of Forward- and Backscattering Signatures on Doppler-Reflectometry Signals
}

Filipe da Silva, Stéphane Heuraux, Evgeniy Gusakov, Alexei Yu Popov

\section{To cite this version:}

Filipe da Silva, Stéphane Heuraux, Evgeniy Gusakov, Alexei Yu Popov. A Numerical Study of Forward- and Backscattering Signatures on Doppler-Reflectometry Signals. IEEE Transactions on Plasma Science, 2010, 38 (9), pp.2144. 10.1109/TPS.2010.2056703 . hal-00521205

\section{HAL Id: hal-00521205 https://hal.science/hal-00521205}

Submitted on 26 Sep 2010

HAL is a multi-disciplinary open access archive for the deposit and dissemination of scientific research documents, whether they are published or not. The documents may come from teaching and research institutions in France or abroad, or from public or private research centers.
L'archive ouverte pluridisciplinaire HAL, est destinée au dépôt et à la diffusion de documents scientifiques de niveau recherche, publiés ou non, émanant des établissements d'enseignement et de recherche français ou étrangers, des laboratoires publics ou privés. 


\title{
A Numerical Study of Forward- and Backscattering Signatures on Doppler-Reflectometry Signals
}

\author{
Filipe da Silva, Stéphane Heuraux, Evgeniy Z. Gusakov, and Alexei Popov
}

\begin{abstract}
In Doppler reflectometry, two scattering processes may be involved, Bragg backscattering and forward scattering. In this paper, a numerical study of both processes is undertaken using a finite-difference time-domain full-wave code with plasma parameters compatible with Tore Supra or Asdex Upgrade Doppler reflectometers. The spectra modeling the plasma turbulence were chosen to isolate forward- and backscattering events, allowing the segregation of the two different signatures and bring forth the different mechanisms intervening in each of the cases. Some numerical precautions needed for this type of simulations are presented. The results obtained are compared with the theoretical models.
\end{abstract}

Index Terms-FDTD, microwave Doppler reflectometry, wave propagation in plasmas, wave scattering.

\section{INTRODUCTION}

A N IMPORTANT development of reflectometry diagnostics is Doppler reflectometry, a technique that is able to convey information on the plasma turbulence rotation from the spectral shift of the return scattered signal [1]-[3]. Two different scattering processes may be involved in Doppler reflectometry. Bragg backscattering, occurring when the turbulence wavenumber $k_{f}$ and the local wavenumber of the injected wave $k(\mathbf{r})$ are resonant: $k_{f}=2 k(\mathbf{r})$, and forward scattering, which becomes dominant for $k_{f}<2 k_{A}$, where $k_{A}$ is the Airy wavenumber [4]. In small and midsize devices with low levels of turbulence, scattering is accepted to be caused by Bragg backscattering in the vicinity of the oblique cutoff [1]-[3]. Nevertheless, under high turbulence conditions or long plasma paths, as occurring in larger machines such as JET, JT 60, or ITER, a distinct process of cumulative forward scattering is expected to appear, contributing to the Doppler frequency shift of the signal, and spread out the probing beam, reducing the wavenumber resolution [5]. The need to understand

Manuscript received November 27, 2009; revised May 7, 2010 and June 23, 2010; accepted June 26, 2010. Date of publication August 16, 2010; date of current version September 10, 2010. This work was supported in part by the European Communities and Instituto Superior Técnico, carried out within the Contract of Association between EURATOM and IST, and in part by the Fundação para a Ciência e Tecnologia in the frame of the Contract of Associated Laboratory.

F. da Silva is with the Associação EURATOM/IST, Instituto de Plasmas e Fusão Nuclear-Laboratório Associado, Instituto Superior Técnico, 1049-001 Lisboa, Portugal.

S. Heuraux is with Institut Jean Lamour, Faculté des Sciences NancyUniversité UMR 7198 CNRS BP 70239, 54506 Vand, France.

E. Z. Gusakov and A. Popov are with Ioffé Polytekhnicheskaya 26, 194021 St. Petersburg, Russia.

Color versions of one or more of the figures in this paper are available online at http://ieeexplore.ieee.org.

Digital Object Identifier 10.1109/TPS.2010.2056703 scattering phenomena in Doppler reflectometry is the origin of several studies. Spatial and wavenumber resolutions have been evaluated analytically and numerically under Born or paraxial approximation [7], [8]. Spatial distribution of the scattering phenomena, which are usually assumed to occur in the cutoff vicinity, is a key issue for diagnostic applications. In case of low turbulence level, when only single scattering is significant, this problem was investigated in the framework of linear theory numerically in [9] and analytically in [8] and [10]. Those studies assumed a small amplitude of the fluctuations, which allows neglecting multiple-scattering contributions to the received signal. However, this approximation can be incorrect in the plasma periphery, where the turbulence amplitude is usually high, or in large plasma devices, where the probingray trajectory is long [8]. This situation of high turbulent density perturbation level, when the multiple forward scattering is dominant, has been investigated analytically [5]. In this paper, a numerical study of both processes is undertaken using a finite-difference time-domain (FDTD) full-wave code. The spectra modeling the plasma turbulence were chosen to isolate forward- and backscattering events allowing the segregation of the two different signatures. The numerical constraints needed to simulate forward scattering, more demanding than those needed for backscattering, are also underlined.

\section{Code And Simulation Setup}

The code used in this work is an ordinary-mode 2-D FDTD full-wave code, which solves Maxwell equations using a Yee scheme

$$
\begin{aligned}
\mu_{0} \varepsilon_{0}\left(\partial_{t} E_{z}\right)_{i, j}^{n} & =\left(\partial_{x} B_{y}-\partial_{y} B_{x}-\mu_{0} J_{z}\right)_{i, j}^{n} \\
\left(\partial_{t} B_{x}\right)_{i, j+1 / 2}^{n+1 / 2} & =-\left(\partial_{y} E_{z}\right)_{i, j+1 / 2}^{n+1 / 2} \\
\left(\partial_{t} B_{y}\right)_{i+1 / 2, j}^{n+1 / 2} & =\left(\partial_{x} E_{z}\right)_{i+1 / 2, j}^{n+1 / 2} .
\end{aligned}
$$

The plasma is modeled through the definition of the electron density $n_{e}(\mathbf{r}, t)$ and coupled to the code-propagation kernel (1) by the equation of the density current

$$
\left(\partial_{t} J_{z}\right)_{i, j}^{n+1 / 2}=\mathrm{e}^{2} / m_{e}\left(n_{e} E_{z}\right)_{i, j}^{n+1 / 2}
$$

Here, we will use a slab base plasma with radial linear density profile $n_{e_{0}}$. A matrix of turbulence $\delta n_{e}$ is calculated and added to $n_{e_{0}}$

$$
n_{e}(\mathbf{r}, t)=n_{e_{0}}(\mathbf{r}, t)+\delta n_{e}(\mathbf{r}, t) .
$$

The total density $n_{e}(\mathbf{r}, t)$ (profile plus turbulence) is fed into (2). The turbulence matrix is defined using an internal model. 
Usually, we use a model of sum of modes, with $\delta n_{e}$ being defined by

$$
\delta n_{e_{T R B}}=\sum_{i=i_{m}}^{i_{M}} \sum_{j=j_{m}}^{j_{M}} A(i, j) \cos \left[k_{x}(i) x+k_{y}(j) y+\varphi(i, j)\right]
$$

where $A(i, j)$ is a chosen amplitude spectrum and $\varphi(i, j)$ is a random generated phase, combining all possible wavenumbers. Simulations were performed using a rectangular grid with dimensions of $L_{x}=150 \lambda_{40 \mathrm{GHz}}$ by $L_{y}=150 \lambda_{40 \mathrm{GHz}}$, which correspond to $3000 \times 3000$ grid points $(112 \times 112 \mathrm{~cm})$, where the Cartesian coordinate dimensions $L_{x}$ and $L_{y}$ correspond to the radial and poloidal dimensions in a tokamak. Part of the box comprises the emission setup (antenna and optical system) and a vacuum distance between the antenna and the plasma. In this work, a monostatic 2-D H-plane horn antenna with a total flare angle of $2 \psi=43.6^{\circ}$ has a half-power beamwidth of $22^{\circ}$. A lens with an effective focal length of $15.7 \lambda_{40 \mathrm{GHz}}(11.8 \mathrm{~cm})$, placed at a distance of $4 \lambda_{40 \mathrm{GHz}}(3 \mathrm{~cm})$ from the antenna mouth, focuses the antenna beam into a low divergence beam with a planar wavefront. The $-3-\mathrm{dB}$ width of the radiation diagram taken at a radius of $50 \lambda_{40 \mathrm{GHz}}$ is $18^{\circ}$. Through the use of a prism, the beam is deflected into the plasma with an injection angle $\alpha=7^{\circ}$ [12]. The antenna and the optical system (lens plus prism) appear on Figs. 2 and 5. The plasma measures $L_{x p}=66 \lambda_{40 \mathrm{GHz}}$ by $L_{y p}=150 \lambda_{40 \mathrm{GHz}}$ or $1353 \times$ 3000 grid points $(50 \mathrm{~cm} \times 112 \mathrm{~cm})$. The probing frequency is $f=40 \mathrm{GHz}$, corresponding to a vacuum wavelength of $\lambda_{0}=$ $0.75 \mathrm{~cm}$ and to a critical density $n_{c} \approx 2 \cdot 10^{19} \mathrm{~m}^{-3}$. Turbulence is homogeneous, and its rms level is defined referring to the critical density for $35 \mathrm{GHz}$ as $\delta n_{e_{T R B}}^{\mathrm{RMS}} / n_{e}\left(x_{c_{35 \mathrm{GHz}}}\right) \times 100 \%$. Calculations have been made for values of $0.1 \%, 0.25 \%, 1.0 \%$, $2.5 \%, 3.0 \%, 5.0 \%, 7.5 \%$, and $10.0 \%$. The plasma flows in the $y$-direction, corresponding to the poloidal direction in a tokamak, with constant velocity $v_{y}=22.5 \times 10^{6} \mathrm{~m} \cdot \mathrm{s}^{-1}$, i.e., $v_{y} / c=7.5 \%$.

\section{Model for Turbulence}

In these simulations, a Kolmogorov-like amplitude $k$ spectrum $A(i, j)$ was used as defined by a flat top up to a chosen wavenumber from where it decays according to a $k^{3}$ rule; in concrete terms

$$
S_{\mathrm{amp}}(k)=\frac{b}{(k-a)^{3}}
$$

where the constants $a$ and $b$ adjust the shape of the decay. We have chosen this amplitude spectrum shape to have a behavior similar to Analyse Localisee du Transport Anormal par diffusion dune lumiere IR (ALTAIR)-French acronym for local analysis of anomalous transport using infrared light)-experimental data, where the amplitude of the fluctuation wavenumber is assumed to have a constant top followed by a decay according to a power law $k_{f}^{3}(f)$ (both along radial and poloidal directions). ALTAIR was an experiment installed at Tore Supra to measure density fluctuations throughout the whole plasma with collective infrared laser scattering [6].

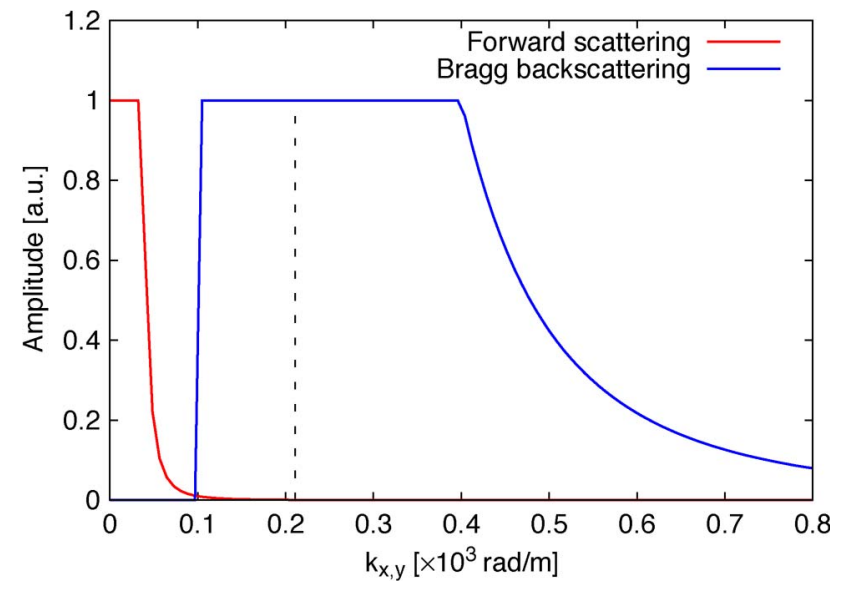

Fig. 1. Representation of the two amplitude spectra used. The Bragg resonant wavenumber $k_{\mathrm{Bragg}}=211.11 \mathrm{rad} \cdot \mathrm{m}^{-1}$ is marked with the broken line.

Calculating the wavenumber value associated with the linear response of the Bragg backscattering at the oblique cutoff, given by $k_{\text {Bragg }}=2 k_{0} \sin (\alpha)$, we have, for the values of frequency and injection angle used, $k_{\mathrm{Bragg}}=211.11 \mathrm{rad} \cdot \mathrm{m}^{-1}$, corresponding to a wavelength $\lambda_{\mathrm{Bragg}}=3.3 \mathrm{~cm}$.

For the first set of simulations, a narrow-width lowwavenumber spectrum was used with a flat top ranging from zero up to $k=37.22 \mathrm{rad} \cdot \mathrm{m}^{-1}$ from where it falls according to the choice of parameters $a=20 \mathrm{rad} \cdot \mathrm{m}^{-1}$ and $b=$ $5.11 \times 10^{3} \mathrm{rad}^{3} \cdot \mathrm{m}^{-3}$. At $k=100 \mathrm{rad} \cdot \mathrm{m}^{-1}$, we have $S=$ 0.01 . It is clear that the wavenumber for the occurrence of linear Bragg backscattering $k_{\text {Bragg }}$ is not present in the spectrum, and therefore, only forward scattering will be present. A second set of simulations was done with a wideband $k$ spectrum with a flat top extending up to $k=400 \mathrm{rad} \cdot \mathrm{m}^{-1}$ and then decaying to $S=0.01$ at $k=1500 \mathrm{rad} \cdot \mathrm{m}^{-1}$, given that $a=97 \mathrm{rad} \cdot \mathrm{m}^{-1}$ and $b=27.6 \times 10^{6} \mathrm{rad}^{-3} \cdot \mathrm{m}^{-3}$. The resonant Bragg wavenumber $k_{\text {Bragg }}$ is now contained in the spectrum, and Bragg backscattering will occur. Additionally, in this second spectrum shape, the low wavenumbers from zero to $k=100 \mathrm{rad} \cdot \mathrm{m}^{-1}$ were removed to prevent the occurrence of forward scattering and isolate the backscattering response. In Fig. 1, the two spectra used are shown. The Bragg resonant wavenumber $k_{\text {Bragg }}=211.11 \mathrm{rad} \cdot \mathrm{m}^{-1}$ is marked with the broken line. It is clear that the narrow amplitude spectrum does not contain wavenumber components to allow Bragg backscattering. The wide spectrum, on the other hand, has them in its flat-top region, while the removal of the low wavenumbers prevents forward scattering.

\section{Simulation Considerations}

One vital aspect to take into account in reflectometry simulations in general, and in Doppler reflectometry in particular, is the injection of the signal. The injection method used defines a radiation diagram which determines the wavenumber vectors injected in the plasma. Ideally, a single wavenumber (a plane wavefront) from a narrow nondivergent beam should be used, assuring that a narrow zone within the plasma is probed with a unique wavenumber. The antenna setup described 
in Section II guarantees this requirements. Another point to take into consideration is the fact that the received scattered signal has a much lower amplitude than the injected one, implying the need for a proper signal decoupling. Without it, the minute received signal will have its characteristics masked by the main emission. It is important that the technique used in the code does not lose the connection with reality; otherwise, the significance of the results will be compromised. For instance, the existence of sidelobes in a radiation diagram and the effect of mode selection in a waveguide are important aspects that influence the response of a reflectometer and must be kept in the simulation setup. Our code addresses the problem of signal injection using a unidirectional transparent source (UTS) associated with an optical system [11], [12]. The UTS allows the injection of the probing wave only in the forward direction and effectively separates the weaker received signal from the main emission. Full-size spatial dimensions can be simulated with the code, but full temporal simulations, due to the small time step discretization dictated by Courant-Friedrichs-Lewy (CFL) conditions, impose the need to scale down the duration of the events simulated and, consequently, to scale up the velocities involved. Let $R_{t}$ be the ratio between $t_{\text {real }}$, the duration of a reflectometry probing (either fixed or swept frequency), and the time simulated $t_{\text {siml }}$

$$
R_{t}=\frac{t_{\text {real }}}{t_{\text {siml }}}
$$

A plasma feature, with some characteristic dimension $L_{p}$, moving with velocity $v_{\text {real }}$ will take a time $T_{\text {real }}$ to pass a given point. In the simulation, this event will be reproduced taking a time

$$
T_{\text {siml }}=\frac{T_{\text {real }}}{R_{t}}
$$

with a corresponding increase in velocity

$$
v_{\text {siml }}=\frac{L_{p}}{T_{\text {siml }}}=R_{t} \frac{L_{p}}{T_{\text {real }}}=R_{t} v_{\text {real }} .
$$

This will also affect the frequency shift due to Doppler effect

$$
\begin{aligned}
\Delta f_{\text {siml }} & =\frac{1}{2 \pi} \mathbf{k}_{p} \cdot \mathbf{v}_{\text {siml }} \\
& =\frac{1}{2 \pi} R_{t} \mathbf{k}_{p} \cdot \mathbf{v}_{\text {real }} \\
& =\frac{1}{2 \pi} R_{t} \Delta f_{\text {real }} .
\end{aligned}
$$

These effects can be fully compensated, the correct real values regained, and the deformation of the shape of the signal spectra recovered, as verified in [11].

Preliminary studies indicate that in the presence of significant forward scattering, to obtain the long time evolution of the system and to give statistical meaning to the results, long time series must be used. This implies the use of large turbulence matrices and, consequently, a large number of iterations. The turbulence matrices used in these simulations have a poloidal dimension of $L_{m}=1500 \lambda_{40 \mathrm{GHz}}(1.12 \mathrm{~m})$, corresponding to 30000 grid points. The number of iterations performed

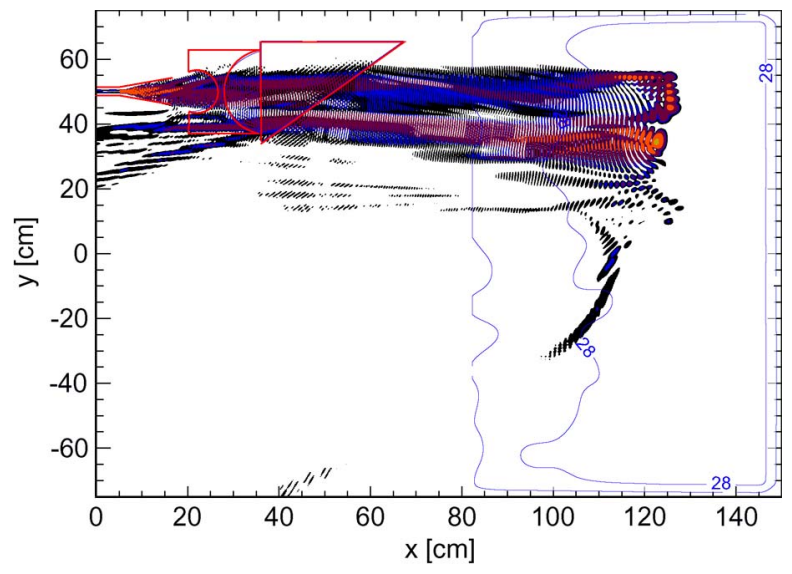

Fig. 2. Snapshot of the electric field obtained with the narrow Kolmogorov spectrum for a level of turbulence of $10 \%$.

was $N_{\text {iter }}=800000$, a value considerably larger than usually observed in reflectometry simulations, a situation due to the very long wavelength associated with forward scattering, which implies a large number of points to obtain significant averages.

\section{FORWARD-SCATTERING SimUlation RESUltS}

A snapshot of the electric-field structure obtained is shown in Fig. 2 for a turbulence level of $10 \%$. Under this strong turbulence level, the beam is split into several subbeams with a loss of coherence and resolution. Near the oblique cutoff, wave trapping can be observed together with multireflections.

The evolution of the scattering process can observed, analyzing the temporal behavior of the signals, for a given turbulence matrix at three levels of turbulence of $0.1 \%, 1 \%$, and $10 \%$. In Fig. 3, the results obtained are summarized. At the top, the scattered signals are shown, and one can notice their intermittent nature and an increase in the amplitude of the bursts with turbulence, which is confirmed by the curves of the windowed rms amplitude of the scattered signals, shown at the bottom, obtained with a sliding window of $\sim 2.5 T_{0}$.

The previous analysis has shown how the behavior of the scattered signals evolves during the simulation, while the following results have been calculated globally on the whole signal, and averages are obtained using eight runs with different realizations of the narrow low-wavenumber $k$-spectrum according to (4). The average rms value of the amplitude of the scattered signals appears in Fig. 4-top (in red) via the turbulence level. Individual rms curves (for each of the runs) are shown in dots. There is an initial linear response of up to $2.5 \%$ followed by a nonlinear saturation. As the turbulence level increases, the dispersion of the individual curve values around the average curve increases. At the bottom of Fig. 4, the eight-run average spectra of the signals are shown. As the turbulence level increases, changes in the behavior of the spectra are observable. There is both an enlargement of the width of the spectra and a displacement of the maxima clearly seen in the $0.1 \%-2.5 \%$ curves, until the limit values are reached for both the enlargement and the maxima. The width of the spectra (at $-3 \mathrm{~dB}$ ) rises from 0.1 up to $0.5 \mathrm{GHz}$. The limit for the maximum deviation from the carrier is the theoretical value 

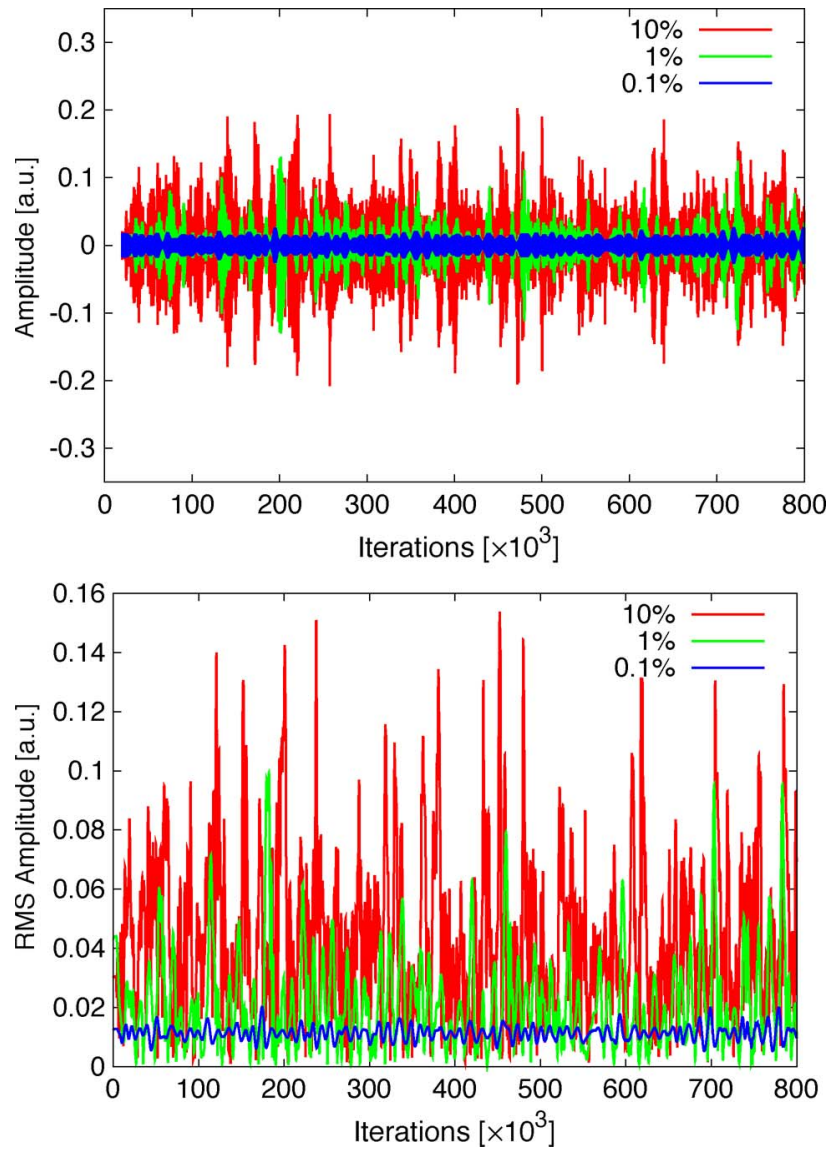

Fig. 3. Results from the narrow Kolmogorov spectrum for three levels of turbulence $(0.1 \%, 1 \%$, and $10 \%)$. (Top) multiscattered returned signal. (Bottom) Windowed rms amplitude of the scattered signals.

for the Bragg backscattering deviation at the oblique cutoff $\Delta f=k_{f} v_{y} / 2 \pi=-0.76 \mathrm{GHz}$, and it is marked in the figure by a black vertical line. In addition to the spectral broadening, for higher turbulence values of 5\%-10.0\%, a nonlinear response appears in the interval of 37-39 GHz. Notice that, for the lowest value of turbulence $(0.1 \%)$, one can still see the very narrow mark of the carrier at $40 \mathrm{GHz}$.

\section{BACKSCATtERING Simulation Results}

A snapshot of the electric-field structure obtained is shown in Fig. 5 for a turbulence level of $10 \%$. When compared with the field structure shown in Fig. 2, we notice a completely different field structure. The power is being scattered in a much finer structure. The structures showing wave trapping are not present.

With the same procedure used in the former section, the results for the wide Kolmogorov spectrum were processed. The same temporal-analysis procedure was repeated, and the results are shown in Fig. 6. The signals show a more continuous nature than the one observed in the previous case. The oscillations in the windowed rms curves have a much higher frequency than the one from the forward-scattering case and show a noneruptive nature of the signal.

The average global behavior was obtained as before for eight runs with different matrix realizations, and the results are shown in Fig. 7. The average rms signal-amplitude curve shows an
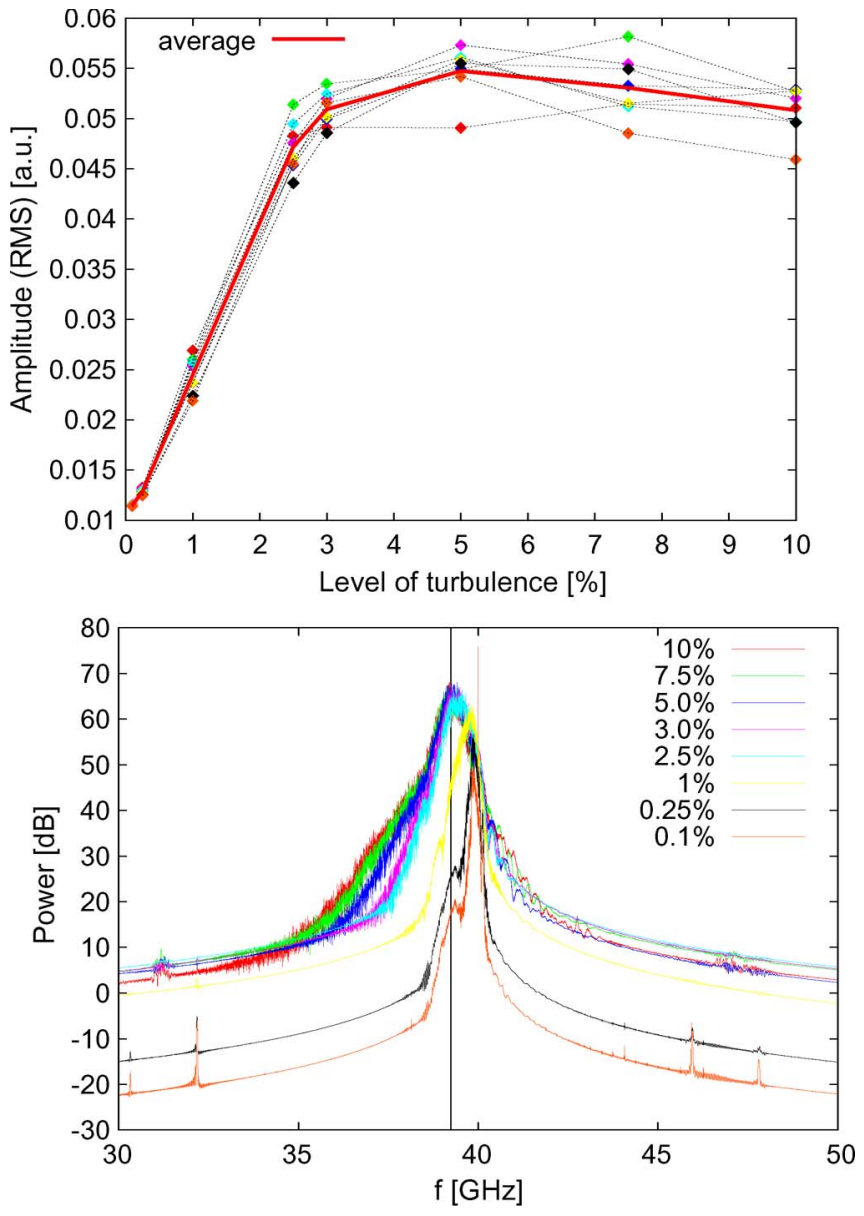

Fig. 4. (Top) RMS amplitude values of the multiscattered returned signals for eight different realizations of narrow Kolmogorov turbulence matrices for several turbulence levels. The continuous curve is the average rms value. (Bottom) Average spectra of the returned signals for different levels of turbulence. Each curve is an average of eight individual spectra.

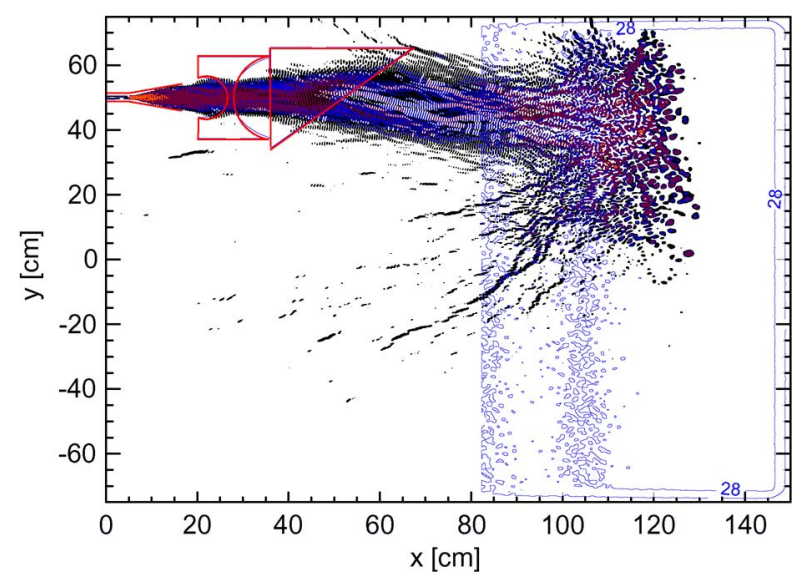

Fig. 5. Snapshot of the electric field obtained with the wide bandpass Kolmogorov spectrum for a level of turbulence of $10 \%$.

initial linear response of up to $\sim 1 \%$, reaching a maximum around $2.5 \%$, from where it starts to decay. The maximum dispersion of the individual rms curves (lesser than the forwardscattering case) is also reached at $2.5 \%$. The average spectrum curves show also a behavior distinct from the former case. All the curves are centered around the Bragg deviation 

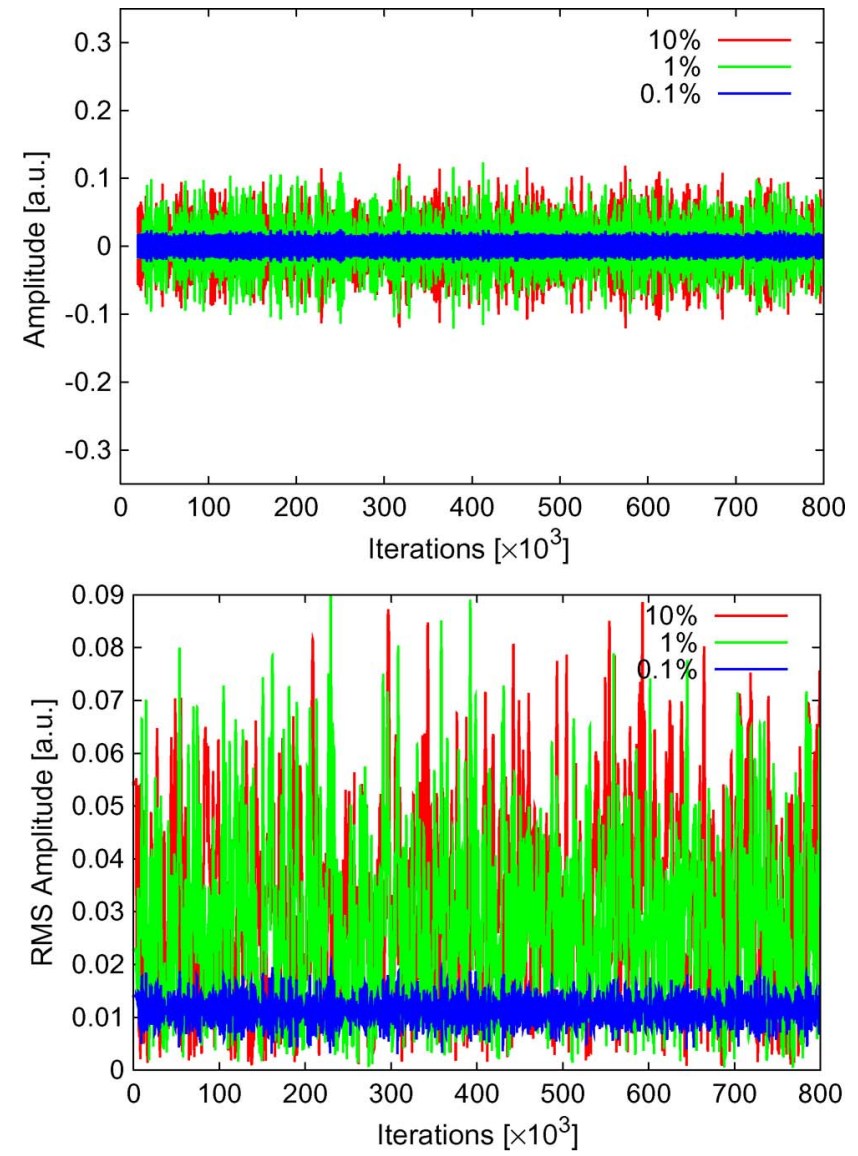

Fig. 6. Results from the wide bandpass Kolmogorov spectrum for three levels of turbulence $(0.1 \%, 1 \%$, and $10 \%)$. (Top) Multiscattered returned signal. (Bottom) Windowed rms amplitude.

$\Delta f=k_{f} v_{y} / 2 \pi$, independent of the level of turbulence. The progressive increase of the width of the spectra seen on the former case is not observed here. The half-power widths are more or less the same, which are $\approx 0.5 \mathrm{GHz}$. For higher levels of turbulence, strong nonlinear effects appear in the interval of $37-39 \mathrm{GHz}$.

\section{DISCUSSION}

The simulations performed illustrate quite clearly the different mechanisms intervening in Bragg backscattering and forward-scattering processes. Forward scattering appears as a process of intermittent nature, resulting from multiple scattering contributions, while the Bragg backscattering has a faster and more continuous character, resulting essentially from a single scattering event in the vicinity of the oblique cutoff. Simulations indicate than the slower nature of forward scattering requires longer simulation time series than the one needed to study backscattering, which is at least ten times longer. The longer is the time series obtained, the closer we will be to the theory which is formulated in an asymptotic limit [5], [13]. The spectral behavior of the scattered signals is distinct in the two situations. For Bragg backscattering, the spectra suffer for all levels of turbulence; the maximum linear Bragg deviation allowed in the system, which is given by $\Delta f=k_{f} v_{y} / 2 \pi$ occurs at the oblique cutoff where the wave radial wavenumber $k_{r}=0$
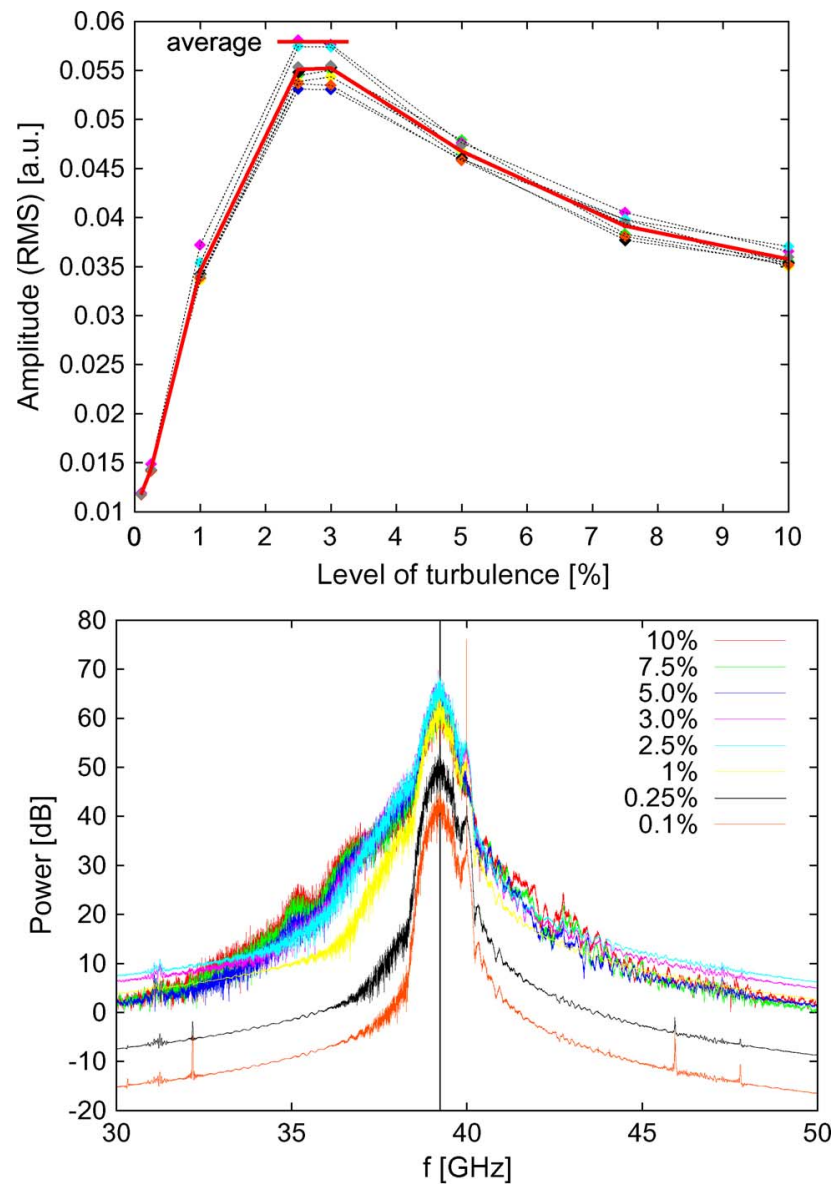

Fig. 7. (Top) RMS amplitude values of the backscattered returned signals for eight different realizations of wide bandpass Kolmogorov turbulence matrices for several turbulence levels. The continuous curve is the average rms value. (Bottom) Average spectra of the returned signals for different levels of turbulence. Each curve is an average of eight individual spectra.

and $k_{f}=2 k_{p}$, with $k_{p}$ being the poloidal wavenumber of the wave. This confirms Bragg backscattering as a phenomenon occurring mainly at the vicinity of the oblique cutoff. The results for forward scattering show an evolution of the Doppler deviation from the carrier to the maximum Bragg deviation together with an enlargement of the peak as the turbulence level increases. This is in agreement with the theoretical models for forward scattering proposed [5], [13]. These models use Gaussian amplitude spectra to model the fluctuations instead of the Kolmogorov-like spectra, which is closer to the experiments used in our model. This, nevertheless, does not seem to play a larger role since the predictions of the model and the results of the simulations are in close agreement. One exception is on the amplitude of the returned forward-scattered signals, which is higher in the simulations for turbulence levels that are higher than 5\%. This, we believe, is due to the fact that in the simulation, there are additional effects that are not included in theory, namely, the appearance of hill-hole zones which trap the wave. These cavities have a fill-relax time that trap the waves over periods much longer than the time of flight. When relaxing, the additional release of electric field contributes to the signal in sudden bursts. Another additional factor outside the domain of the theory, verified in simulations, is the emergence of secondary cutoff surfaces, sometimes detached from 
the location of the main cutoff surfaces. For the same levels of turbulence $>5 \%$, the beam, in the case of Bragg backscattering near the oblique cutoff, dispersed in all directions, justifying the reduction of the rms power observed. Also observed in the simulations is the appearance of nonlinear secondary responses for turbulence above $5 \%$, which is of lower power than the main, having a Doppler displacement higher than the one given by the Bragg rule and which can be justified by a the nonlinear Bragg response, as described by the second unstable zone of Mathieu's equation.

\section{ACKNOWLEDGMENT}

The views and opinions expressed herein do not necessarily reflect those of the European Commission, IST, and FCT.

\section{REFERENCES}

[1] G. D. Conway, J. Schirmer, S. Klenge, W. Suttrop, and E. Holzhauer, and ASDEX Upgrade Team, "Plasma rotation profile measurements using Doppler reflectometry," Plasma Phys. Control. Fusion, vol. 46, no. 6, p. 951, Apr. 2004.

[2] P. Hennequin, C. Honor, A. Truc, A. Qumneur, N. Lemoine, J.-M. Chareau, and R. Sabot, "Doppler backscattering system for measuring fluctuations and their perpendicular velocity on Tore Supra," Rev. Sci. Instrum., vol. 75, no. 10, pp. 3881-3883, Oct. 2004.

[3] M. Hirsch and E. Holzhauer, "Doppler reflectometry with optimized temporal resolution for the measurement of turbulence and its propagation velocity," Plasma Phys. Control. Fusion, vol. 46, no. 4, p. 593, Mar. 2004.

[4] C. Fanack, I. Boucher, S. Heuraux, G. Leclert, F. Clairet, and X. Zou, "Ordinary-mode reflectometry: Modification of the scattering and cutoff responses due to the shape of localized density fluctuations," Plasma Phys. Control. Fusion, vol. 38, no. 11, p. 1915, Nov. 1996.

[5] E. Z. Gusakov, A. V. Surkov, and A. Y. Popov, "Multiple scattering effect in Doppler reflectometry," Plasma Phys. Control. Fusion, vol. 47, no. 7, p. 959, May 2005.

[6] P. Devynck, X. Garbet, C. Laviron, J. Payan, S. K. Saha, F. Gervais, P. Hennequin, A. Quemeneur, and A. Truc, "Localized measurements of turbulence in the TORE SUPRA tokamak," Plasma Phys. Control. Fusion, vol. 35, no. 1, p. 63, Jan. 1993.

[7] V. Bulanin and M. Yafanov, "Spatial and spectral resolution of the plasma Doppler reflectometry," Plasma Phys. Rep., vol. 31, no. 1, pp. 47-55, Jan. 2006.

[8] E. Z. Gusakov and A. V. Surkov, "Spatial and wavenumber resolution of Doppler reflectometry," Plasma Phys. Control. Fusion, vol. 46, no. 7, p. 1143, Jun. 2004.

[9] M. Hirsch, E. Holzhauer, J. Baldzuhn, B. Kurzan, and B. Scott, "Doppler reflectometry for the investigation of propagating density perturbations," Plasma Phys. Control. Fusion, vol. 43, no. 12, p. 1641, Oct. 2001.

[10] E. Gusakov and B. Yakovlev, "Two dimensional theory of fluctuation reflectometry diagnostics," in Proc. 28th EPS Conf. Control. Fusion Plasma Phys., Funchal, Portugal, Jun. 18-22, 2001, vol. 25A, p. P1.093.
[11] F. da Silva, S. Heuraux, and M. Manso, "Studies on O-mode reflectometry spectra simulations with velocity shear layer," Nucl. Fusion, vol. 46, no. 9, p. S816, Aug. 2006.

[12] F. da Silva, S. Heuraux, and M. Manso, "Unidirectional transparent signal injection in finite-difference time-domain electromagnetic codes-Application to reflectometry simulations," J. Comput. Phys., vol. 203, no. 2, pp. 467-492, Mar. 2005.

[13] A. V. Surkov, "Nonlinear regime of Doppler reflectometry in cylindrical plasmas," Plasma Phys. Control. Fusion, vol. 48, no. 7, p. 901, May 2006.

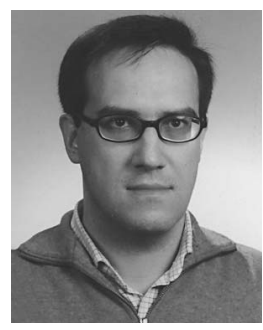

Filipe da Silva received the B.S. degree in electrical and computer engineering from Technical Superior Institute (IST), Lisbon, Portugal, in 1991, the M.S. degree in management of science and technology from the Superior Institute of Economy and Management, Lisbon, and the Ph.D. degree in physics from IST in 2007.

$\mathrm{He}$ is currently with the Institute of Plasmas and Nuclear Fusion, IST.

Dr. da Silva is a member of the Portuguese Engineers Association (Ordem dos Engenheiros).

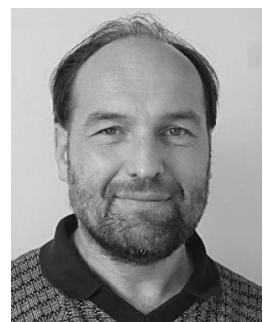

Stéphane Heuraux was born in Epinal, France on May 24, 1958. He received the Ph.D. degree in plasma physics and the "Habilitation à Diriger les Recherches" from the Henri Poincaré University, Nancy, France, in 1986 and 1999, respectively.

In 1983, he was with LPMIA, Henri Poincaré University. He was invited by the CEA Cadarache in 2003-2004 to work on antenna-plasma coupling and reflectometry diagnostic. Since 2007, he has been a Full Professor with the Henri Poincaré University. His scientific interest mainly involves wave in plasma physics, theory, simulation and applications, particularly regarding ponderomotive force, propagation in fluctuating plasmas, whether magnetized or not, reflectometry diagnostics for characterization of density fluctuations in fusion plasmas, antenna-plasma coupling for fusion plasma heating, and RF sheath physics to explain and look ahead of hot spots in fusion devices during RF heating.

Evgeniy Z. Gusakov is with the Division of Plasma Physics, Atomic Physics, and Astrophysics, IOFFE Physical Technical Institute, St. Petersburg, Russia, where he is the Head of the High Temperature Plasma Physics Laboratory. His interests include wave propagation in inhomogeneous turbulent plasmas, nonlinear wave-plasma interaction, RF heating, and microwave plasma diagnostics.

Alexei Popov is with the Division of Plasma Physics, Atomic Physics, and Astrophysics, IOFFE Physical Technical Institute, St. Petersburg, Russia, where he is a Member of the High Temperature Plasma Physics Laboratory. His interests include plasma reflectometry and ECE emission. 